\title{
Research on Stealthy Headphone Detector Based on Geomagnetic Sensor
}

\author{
Ya Liu', Si Chen²and Zhihui Luo ${ }^{1, a}$, Liqing Pan ${ }^{1}$ \\ ${ }^{1}$ Research Institute for Magneto-electronics \& nT Magnetic Field Detection, China Three Gorges University, Yichang 443002, China \\ ${ }^{2}$ College of Science, China Three Gorges University, Yichang 443002, China
}

\begin{abstract}
A kind of stealth headphone detector based on geomagnetic sensor has been developed to deal with the stealth headphones which are small, extremely stealthy and hard to detect. The U.S. PNI geomagnetic sensor is chosen to obtain magnetic field considering the strong magnetic performance of stealth headphones. The earth's magnetic field at the geomagnetic sensor is eliminated by difference between two geomagnetic sensors, and then weak variations of magnetic field is detected. STM8S103K2 is chosen as the central controlling chip, which is connected to LED, buzzer and LCD 1602. As shown by the experimental results, the probe is not liable to damage by the magnetic field and the developed device has high sensitivity, low False Positive Rate (FAR) and satisfactory reliability.
\end{abstract}

\section{Introduction}

Stealthy headphone is used as a common tool for cheating and illegal monitoring at present and has brought great trouble to the inspection work. As shown in Figure 1, stealthy headphone is characterized by small size, light weight, easy to use and so on ${ }^{[1]}$. Stealth headphone is mainly divided into two types, namely rare earth type and electronic type ${ }^{[2]}$. The rare earth type is mainly composed of ndfeb magnets, and the vibrations coming from the audio frequency magnetic field enable the wearer to hear sounds. The electronic type mainly relies on coil of inductive electromotive force in the audio field, and the active ceramic drives trumpet to make sounds ${ }^{[3]}$.

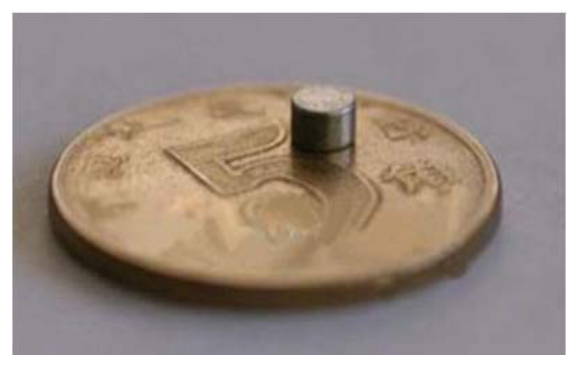

Figure 1. The Surface of Stealthy Headphone.

For each type of stealthy headphone, its internal contains magnetic material, whose near magnetic field strength is about $5 \sim 10 \mathrm{Gs}$. But when the detect distance is $8 \mathrm{~cm}$, the magnetic field strength is attenuated to be below $10^{-3} \mathrm{Gs}$ (the geomagnetic field strength is about $\left.0.5 \mathrm{Gs}\right)^{[3]}$. The main methods for detecting stealth headset are as follows:
1) Visual inspection. The inspector observes whether there is obvious foreign body inside the candidate's ears through naked eye. Because the volume of the stealthy headset which can be stuffed into the external auditory canal directly is smaller and smaller, it is hard for the inspector to find the rice type stealthy headset through visual method.

2) Using electromagnetic wave detection device. Though traditional electromagnetic detection equipment can detect electromagnetic wave, the detection device fails to effectively detect when the carriers don't open the headphone $\mathrm{e}^{[4,5]}$.

3) Using metal detectors. Metal detectors which are sensitive to metal materials with certain volume are easy to lead misinformation when influenced by irrelevant metal, such as belt buckle, key, hairpin and zipper, which are always on the object to be inspected. In addition, it is hard for the metal detector to detect the tiny devices, let alone the rice granular stealthy headphone ${ }^{[5]}$.

In this paper, the U.S. PNI geomagnetic sensor is chosen to obtain magnetic field considering the strong magnetic performance of stealth headphones, the magnetic field is eliminated by the magnetic field of the geomagnetic sensor to obtain the weak magnetic field ${ }^{[6]}$. As shown in Table 1, PNI geomagnetic sensor has many characteristics, this is, small size, low power consumption, high resolution and low cost. The device can effectively detect changes of the magnetic field and eliminate the magnetic field interference. In general, it has good application prospect.

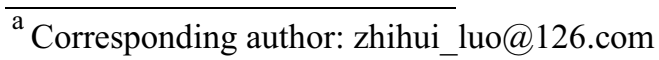




\section{Table 1. Sen-XY-f Characteristic}

\begin{tabular}{|c|c|c|c|c|}
\hline Power Consumption & Package Size & Measuring Range & DIP & Temperature Range \\
\hline $3.3 \mathrm{VDC}, \mathrm{i}<100 \mu \mathrm{A}$ & $6.0 \times 2.1 \times 2.1 \mathrm{~mm}(\mathrm{XY})$ & $\pm 1100 \mu \mathrm{T}( \pm 11 \mathrm{Gs})$ & $13 \mathrm{nT}(0.13 \mathrm{mGs})$ & -40 to $85^{\circ} \mathrm{C}$ \\
\hline
\end{tabular}

\section{Principle of geomagnetic sensor}

The operating circuit of magnetic sensor PNI includes LR oscillation measured positively and reversely which consists of the bias resistors, Schmitt trigger and inductive sensors.

As shown in Figure2, when the circuit works, the Schmitt trigger outputs high voltage level and the inductance sensor and resistor connect the earth, then the positively measure begins to work ${ }^{[7]}$. The partial pressure on resistor keeps increasing, the trigger turns back when the voltage at point $\mathrm{A}$ is higher than the threshold voltage of Schmidt trigger.

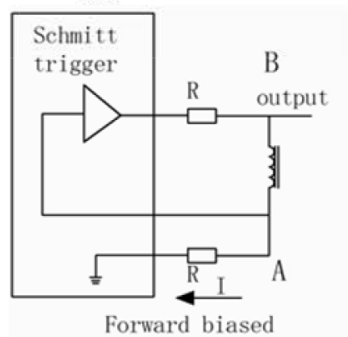

Figure 2. LR Oscillator Circuit Biasing Diagram

The energy storage of the inductance sensor begins to release, the voltage at point A decreases to low voltage level. According to the above steps repetitively, the oscillation circuit based on LR is formed. The period of oscillation directly depends on the size of the resistance and inductance. The process of the reversely measured is similar to the positively measured, in which the current flows through the sensors in the opposite direction. The period of output signal at Point B will be tested by dedicated IC in digital way ${ }^{\left[{ }^{[8]}\right.}$.

When the inductance value of inductive sensors is affected by external magnetic field, the oscillation signal cycle at the output point will change. For a specific sensor, the inductance value is directly decided by the total magnetic field strength. The size of the total magnetic field is

$$
H=k_{o} I+H_{E}
$$

Here $H_{E}$ is the external magnetic fields, $H$ is magnetic field generated by electric current $I$, and $k_{o}$ is a constant which is determined by the sensor material ${ }^{[9,10]}$.

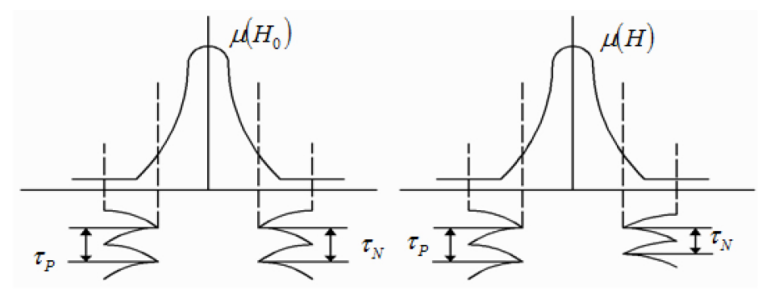

Figure 3. The Oscillation Cycle at Point A
When the external magnetic field is zero, the inductance value of the inductive sensor depends on the magnitude of the current. The oscillation period at point $\mathrm{A}$ for the positive and reverse measurements is the same. As shown in the Figure 3, when the sensor is influenced by the external magnetic field, positive total magnetic intensity increases. The oscillation period at point A $\tau_{N}$ increases when positively measured and decreases when reversely measured. By the differential treatment to the positive and negative oscillation period, the influence of the sensor's own magnetic field can be eliminated and the digital signal proportional to the external magnetic field $H_{E}$ can be obtained.

\section{Hardware design}

Figure 4 shows the hardware structure of the detection device based on the magnetic sensor. STM8S103K2 is selected as the core control chip, of which the SPI interface, UART interface, multiple IO ports and other resources are used.

In the design, the power module consists of power whose control chip is $5 \mathrm{~V}$ and $\mathrm{DC}$ is $3.3 \mathrm{~V}$. $5 \mathrm{~V}$ supplies to control chip STM8 $103 \mathrm{~K} 2$ and $3.3 \mathrm{~V}$ supplies to a magnetic sensor driver chip. The control chip STM8S103K2 and magnetic sensor driver chip MagI2C communicate through SPI interface. Interface RS232 is equipped with voltage conversion chip MAX232, STM8S103K2 downloads program through the serial ${ }^{[1]}$. Stealthy headphone detection device based on terrestrial magnetic sensor uses differential probe circuit to eliminate the influence of magnetic field, the interval of the two SEN-XY-f arranged on the PCB board is $1.5 \mathrm{~cm}, 1 \mathrm{~cm}$ is obtained by experiment. Geomagnetic sensor driver chip MagI2C converts analog magnetic field signal into digital pulse signal, which then passed to the chip STM8S103K2. The threshold of buzzer which controls the buzzer chirping is $500 \mathrm{nT}$, as obtained through the experiment. Set different threshold of LED to control the corresponding LED light, as the measuring result increases through the control process, the number of lighted LED will also increase, different results of the two signal are showed on LCD1602 and presented accurately to inspectors in real time.

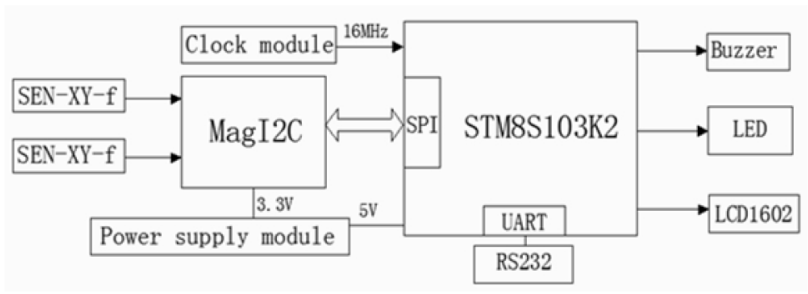

Figure 4.Hardware Structure of the Detection Device 
A Sen-XY-f sensor coil serves as the inductive element in a simple LR relaxation oscillation circuit which is composed of an external bias resistor along with digital gates and a comparator internal to the MagI2C. The coil's inductance is a function of the magnetic field strength. As such, the frequency of the circuit oscillation changes with the strength of the total magnetic field parallel to the sensor. To make a measurement, one side of the coil is grounded while the other side is alternately driven with positive and negative current through the oscillator. The circuit is driven for a set number of circuit oscillations, and the time to complete the oscillations is measured using the MagI2C's internal high-speed clock $^{[12]}$. The MagI2C next switches the bias connection to the coil and makes another measurement. The side previously grounded is now charged or discharged while the other is grounded.

\section{Experiment and Discussion}

Consider that the stealthy headphone will be placed on external auditory canal, the length of adult external auditory canal is about $2.0 \sim 2.5 \mathrm{~cm}$. When inspectors hold stealth headphone detection device, the distance to the human ear is usually in the range of $2.0 \sim 4.0 \mathrm{~cm}$. Magnet generates magnetic field in space which attenuates in accordance with the following equation ${ }^{[8]}$.

$$
H_{0}=K\left(\frac{L+d}{L}\right)^{3} H
$$

To test the magnetic field $H_{0}$ on the surface of the magnet, $H$ is the magnetic field measured by probe. In this design, the measured value is observed by changing the interval of two SEN-XY-f, this is the change of the two SEN-XY-f differential component. Make a contrast experiment to the stealth headset and magnetic earrings.

As shown Figure 5.a. and 5.b, the $\mathrm{X}$ axis presents the distance between the first magnetic sensor and stealth headset, $Y$ axis presents the magnetic field strength. Each of the three figures shows the cases when the distance between the two sensors is $1 \mathrm{~cm}, 1.5 \mathrm{~cm}$ and $2 \mathrm{~cm}$, respectively. In the experiment, the measuring distance is $4.0 \sim 6.5 \mathrm{~cm}$, the magnetic field distribution is contrasted through changing the interval of two sensors. As shown in figure 5.a, two SEN-XY-f are presented. When the measuring distance is fixed, the measured values increases as the interval increases. With the decrease of measuring distance, the measured value of the magnetic field presents growth law of third power. When the value of interval is $2 \mathrm{~cm}$ and the measuring distance is $4.0 \sim 6.5 \mathrm{~cm}$, the measured results for the earrings with magnetic will be more than 500nT, this case leads to misjudgment. When the value of interval is $1 \mathrm{~cm}$ and the measuring distance is $6.5 \mathrm{~cm}$, the measured results for stealth headset will be less than $500 \mathrm{nT}$, the headset fails to be detected in this case. When the value of interval is $1.5 \mathrm{~cm}$ and the threshold is $500 \mathrm{nT}$, the above problems can be effectively solved.

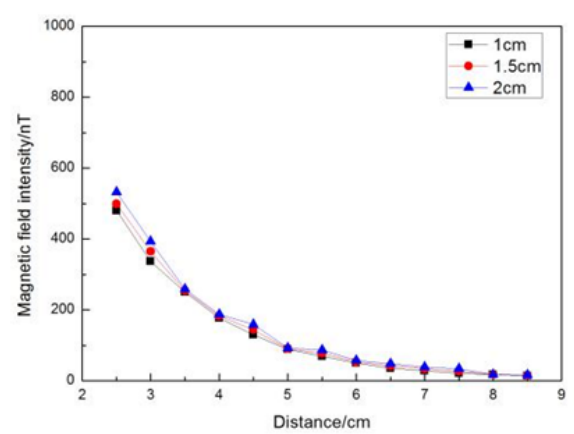

Figure 5.a. Measured Results for Earrings.

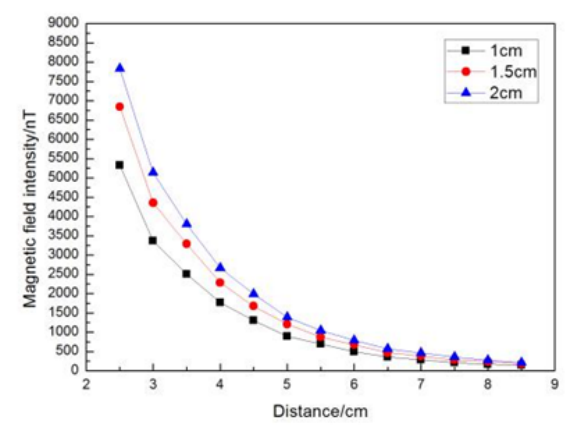

Figure 5.b. Measured Results for Stealthy headphone.

\section{Conclusions}

As shown by the experimental results, the developed device owns high sensitivity, low False Positive Rate (FAR) and satisfactory reliability, has a probe that's not liable to damage by the magnetic field, and is small in size, which has a very good application prospect.

\section{Acknowledgment}

This work is supported by the Graduate Student Research Innovation Foundation of China Three Gorges University (NO. 2015CX126) and Key Technology Support Program of Hubei Province, China (Grant No: 2014BEC060).

\section{References}

1. XuHuanhuan, SongShilei.Exploration on the mechanism of anti cheating in Higher Education.Hubei entrance examination, 36(2012)

2. YangHanling.Talking about the examination of anti cheating equipment.Modern educational equipment in China, 04(2012)

3. WangYun.Stealth headset wits electronic test cheating.China Quality Miles, 10(2006)

4. ZhaoYantian. Modern anti cheating equipment in the examination room. China anti fake Report. 07(2009)

5. ZhangBing, JingYan. Development and application of invisible earphone detector. China Radio, 06(2011) 
6. LiuYa, LuoZhihui. Three axis weak magnetic field detection system based on LabVIEW. Transducer and Microsystem Technologies, 07(2015)

7. ChenYongzhi, ZhangWei. Schmitt trigger and its sensor experiment for example,Middle school physics teaching reference, 11(2015)

8. ZhuBo, YuQi, NingNing. New type of electric reset circuiton low power consumption, Microelectronics, 01(2013)

9. Cirillo, Carla, García-Santiago, Antoni, Joan Manel Hernandez. Magnetic properties of double exchange biased diluted magnetic alloy ferromagnet antiferromagnet trilayers. Journal of Physics: Condensed Matter, 25, 17(2013)

10. V.R.Skal' skii,B.P.Klim,E.P.Pochapskii.Distribution of the induction of a quasi-stationary magnetic field created in a ferromagnet by an attachable electromagnet. Russian Journal of Nondestructive Testing, 48, 1(2012)

11. YanAijun, FangHaiming, ZhouYun. Touch button design based on single chip microcomputer STM8S103 and ST05A. Chemical Defence on Ships, 1(2013)

12. LuLingjie, QingHuibing. Design of intelligent home lighting system based on STM8S103 and WIFI. electric automatization, 03(2014) 